\title{
An Automated Psychoacoustic Testing Apparatus for Use in Cats
}

Yuri B. Benovitski ${ }^{1,2}$, Peter J. Blamey ${ }^{1,3}$, Graeme D. Rathbone ${ }^{1,2}$, James B. Fallon ${ }^{1,3,4}$

${ }^{1}$ Bionics Institute, ${ }^{2}$ Department of Electronic Engineering, La Trobe University, ${ }^{3}$ Department of Medical Bionics, University of Melbourne, ${ }^{4}$ Department of Otolaryngology, University of Melbourne.

Correspondence concerning this article should be addressed to James B. Fallon, Bionics Institute, 384-388 Albert St, East Melbourne, VIC - 3002, Australia. Tel.: +61 39288 3686; fax: +61 392882998

Email: jfallon@bionicsinstitute.org

\section{Abstract}

Animal behavioral studies make a significant contribution to hearing research and provide vital information which is not available from human subjects. Animal psychoacoustics is usually extremely time consuming and labor intensive; in addition, animals may become stressed, especially if restraints or negative reinforcers such as electric shocks are used. We present a novel behavioral experimental system that was developed to allow efficient animal training in response to acoustic stimuli. Cats were required to perform a relatively simple task of moving toward and away from the device depending on whether the members of a tone pair were different or the same in frequency (go/no-go task). The experimental setup proved to be effective, with all animals $(\mathrm{N}=7)$ performing at above $90 \%$ correct on an easy task. Animals were trained within 2 to 4 weeks and then generated a total of 150 to 200 trials per day, distributed within approximately 8 self initiated sessions. Data collected using this system were stable over 1 week and repeatable over long test periods (14 weeks). Measured frequency discrimination thresholds from 3 animals at 3 different reference frequencies were comparable with previously published results. The main advantages of the system are: relatively simple setup; large amounts of data can be generated without the need of researcher supervision; multiple animals can be tested simultaneously without removal from home pens; and no electric shocks or restraints are required.

Abbreviations: ABR, auditory brain response; SPL, sound pressure level; CR, correct rejection; FA, false alarm; d', d-prime; $\Delta \mathrm{f}$, frequency difference; $\Delta \mathrm{f} / \mathrm{f}$, ratio between the frequency difference and reference frequency. 
Keywords: Psychoacoustic, behavior, cat, hearing, frequency discrimination 


\section{Introduction}

Animal psychoacoustics are often used to study the mammalian auditory system; however, they can be prohibitively time consuming (e.g. Sloan et al., 2009) and often only some of the animals can learn the task with satisfactory performance levels. Additionally, behavioral response thresholds often differ between different experimental setups, as a result of variations in task complexity, animal response biases, or stress levels caused by different testing paradigms.

Previously reported methods of behavioral testing usually involve animal restraint (e.g. Brown et al., 2004, May et al., 1995, Smith et al., 1994), food deprivation prior to the experiment (e.g. Koot et al., 2009, Liu et al., 2010, Sloan et al., 2009) and/or negative reinforcement such as electric shocks (e.g. Beitel et al., 2000, Elliott et al., 1960, Heffner and Heffner, 1985, Vollmer et al., 2001). Additionally, most of the reported procedures require removal of the animal from its usual living cage (home cage) to a special laboratory, which has been shown to impose additional stress on the animals (Elliott et al., 1960, Carlstead et al., 1993, Duke et al., 2001, Balcombe et al., 2004). Increased stress is associated with impaired memory, attention, and locomotor activity (Ammersdörfer et al., 2012), all of which are likely to impact performance. To address the issues mentioned above, we developed a new behavioral testing paradigm to allow unsupervised training of animals to respond to acoustic stimuli by moving back and forth from the stimulus source (a go/no-go task) in their home cage. The paradigm required the development of a testing rig that includes a food delivery mechanism which presents positive reinforcement for correct behavior. Apart from short timeout periods following an incorrect response, the procedure does not require any negative reinforcers. If run continuously in the animal's home cage, the device can act as the animal's main, or sole, food source. The ability to perform behavioral testing in the animal's home cage leads to fast training with minimal disruption to animal's normal routine.

To test the system we measured frequency discrimination ability, since it is a widely used hearing test (Wilson and Dorman, 2008). Frequency discrimination thresholds can be easily determined with human subjects, as they are able to respond and indicate what they hear (Clark, 1998, Pretorius and Hanekom, 2008). Conducting the same experiment on cats is harder and requires a procedure which can produce sufficient amounts of stable and repeatable data. Frequency discrimination thresholds in normal hearing cats have been reported in a number of previous studies (Brown et al., 2004, Hienz et al., 1993, Elliott et al., 
1960) and we compared performance measured using our testing paradigm with that reported in those studies.

\section{System description}

\subsection{Apparatus}

The behavioral experimental setup involved training cats to respond to acoustic stimuli using food as a positive reinforcer. The main components of the behavioral system are shown in Figure 1. They were: 1. Vifa Vline tweeter which can deliver a sound pressure level (SPL) above $80 \mathrm{~dB}$ over a wide frequency range (0.2 to $40 \mathrm{kHz}) ; 2$. Digitech $5 \mathrm{~W}$ RMS audio amplifier with total harmonic distortion of $0.5 \%$ and signal-to noise-ratio of $79 \mathrm{~dB} ; 3$. Welco WP1000 peristaltic pump; 4. Two light emitting diodes; 5. Food tank with stirrer; 6. Sharp optoelectronic proximity sensor (Sharp-2y0a02); and 7. National Instruments (USB-6211) data acquisition device to control the various components and connect to a personal computer which acted as interface and data storage element of the system. The cat was required to perform the relatively simple task of moving toward and away from the food source while its location with respect to the machine was monitored by the proximity sensor. The three different sensing distances, required by the training procedure (section 2.2), were determined by a single sensing device located in front of cat's head. The size of the unit is $14 \times 27 \times 35 \mathrm{~cm}$. It can be constructed for about $\$ 250$ in components and a number of units can be controlled simultaneously by a single computer and multiple data acquisition devices.

\subsection{Procedure}

A go/no-go (same-different) paradigm was used to train the animals (Tong et al., 1982). The flowchart diagram (figure 2) maps out the cycle of a standard trial. The animal initiated a trial by moving towards the food source (passing sensing distance 1) and staying in that position for at least 1 second. A trial initiation stimulus (illumination of a blue light) was then presented, and the animal was required to move away from the device (pass sensing distance 2), in order for the test to begin. The system then randomly presented a pair of same or different frequency tones (similar to Recanzone et al., 1991). Each pair was presented 3 to 5 times unless the animal responded sooner. The different-frequency pairs were target stimuli, to which the animal was required to respond by moving toward the bowl (passing sensing distance 3 ) to receive its 
reward. The same-frequency pairs were the non-target stimuli, in response to which the animal was required to stay away from the device.

A hit (animal approached the bowl during a target stimulus pair) was followed by the pump rotating for a maximum time of 2 seconds, delivering 2 to 3 grams of food. Critically, the pump stopped running if the animal moved away from the bowl, meaning food would not be dispensed while the animal was absent. A correct rejection $(\mathrm{CR})$ was recorded if same-frequency pairs were presented without an approach from the animal, after which a new trial could be activated with no delay if the animal approached the food source again. A false alarm (FA) (approaching the food source after presentation of a same-frequency pair) was followed by a 30 second penalty timeout, during which a green light was presented and a new trial could not be initiated. A miss (failing to approach after presentation of a different-frequency pair) was followed by 0 to 6 seconds of penalty time out without any light indication.

\subsection{Acoustic stimuli and loudness roving}

For the frequency discrimination task, tone pips of $195 \mathrm{~ms}$ with 4.3-ms rising edge and 49-ms falling edge were used. The interval between the two tones within a pair was $750 \mathrm{~ms}$, and that between two pairs was $2350 \mathrm{~ms}$. The stimulus level was set to $80 \mathrm{~dB}$ SPL which was always audible (i.e. higher than auditory brain response (ABR) threshold). SPL variability at 3 different locations around the speaker, where the animal's head was usually positioned during tone presentation, was less than $5 \mathrm{~dB}$.

It is important to establish that the stimuli are discriminated solely on the basis of the parameter of interest, in this case frequency, and not on the basis of other cues, such as a SPL difference between the two presented tones. Therefore, level roving of $\pm 5 \mathrm{~dB}$ SPL was used on both tones, to avoid level cues. Absence of level cues was confirmed using post-hoc analysis of the data (i.e. by comparing hit rate for lower dB SPLlow frequency, higher dB SPL-high frequency pairs versus lower dB SPL-high frequency, higher dB SPLlow frequency pairs (see section 3.7)).

\section{Evaluation}

\subsection{Subjects and Environment}

Seven cats were used to evaluate our method, of which 3 were used to establish frequency discrimination 
thresholds. All procedures were approved by the Royal Victorian Eye and Ear Hospital Animal Ethics Committee (project number 10/207AB). Animal weights were regularly monitored to ensure sufficient food intake at all times. All animals in this experiment were exposed to the usual sounds associated with a normal animal facility, including their own vocalizations and those of other cats housed in the same room. Sounds other than cat vocalizations were typically below $2 \mathrm{kHz}$ and below $60 \mathrm{~dB}$ SPL (Fallon et al., 2009) whereas testing was conducted using 3,6 , and $8 \mathrm{kHz}$ at around $80 \mathrm{~dB}$ SPL.

\subsection{Training}

Training commenced with an adaptation phase (similar to Ernst et al., 2005) during which no same frequency pairs were presented (i.e. the animal was required to approach and be rewarded every time a sound was presented). Therefore, unless the animal gave up and stopped accessing the device, it received sufficient amounts of food and the machine was established as the main food source. Same frequency pairs (non-target stimuli) were gradually added within 1-2 weeks to make half of all trials. Eventually, animals were trained to differentiate between two similar (low-low and high-high) and two different (high-low, lowhigh) pairs of stimuli.

To measure performance, we used signal detection theory and considered d-prime (d') as an unbiased performance measure. $\mathrm{d}^{\prime}=\mathrm{z}($ hit rate $)-\mathrm{z}($ FA rate $)$ where $\mathrm{z}$ is the inverse cumulative normal distribution with a mean of 0 and standard deviation of 1 (Heeger, 1997).

A typical learning curve during the training stage is presented in Figure 3C. Prior to day 5, only target (different-frequency pairs) stimuli were given, so it was not possible to determine d'. On day 8 the animal generated above 100 trials (figure 3A) as it began to associate food rewards with approaching the test apparatus. Percent of non-target stimuli gradually increased from day 1 to 10 and then fixed at 50\%. Up to day 25 , hit and FA rates were similar (figure $3 B$ ), indicating poor performance $\left(d^{\prime}<1\right)$. After 30 days there was a clear separation between hit and FA rates, indicating an increase in performance. Average d' of the last 7 measurements was 2.45 (linear trend slope of 0.008 ), which is equivalent to approximately $85 \%$ correct responses. Behavioral performance of all tested animals is summarized in table 1.

\subsection{Trial selection}


Some variability in animal behavior is expected, and some trials may be less informative than others (e.g., trials associated with accidental activation of the system). To address this issue, trial rate (i.e., the inverse of the time interval between two consecutive trials) was monitored. Animals activated trials in clusters as illustrated in figure 4. To help eliminate less informative trials when the animal might not be fully engaged in the discrimination task, a test session was defined as more than 5 consecutive trials that were less than 5 minutes apart (0.003 trials/s, dashed line Figure 4). Empty circles in figure 4 indicate 'out-of-session' trials, which were excluded from further analysis, while filled circles indicate 'in-session' trials that were used in subsequent data analysis. The rationale for discarding 'out-of-session' trials is based on the observation that animals occasionally initiated trials accidentally while moving around their cage, rather than to be fed. It was assumed that on such trials, the animal did not engage in the task, and those trials were therefore not considered when determining performance. On average, $18.7 \%$ of all trials were out-of-session. Ratios of inand out-of-session trials for individual animals are shown in Table 1.

The procedure requires that each trial be initiated by the animal, and thus the number of trials per session and number of sessions per day varied between animals. After training, animals initiated on average 183 trials within 8.5 sessions per day, with the lowest number of trials per session being 6 , as defined by the protocol, and the average being 17. Performance of all out-of-session trials was usually lower than performance within sessions.

\subsection{Frequency difference threshold}

To assess the system we measured frequency discrimination thresholds for 3 cats (A1, A2, and A3). A manual frequency difference $(\Delta \mathrm{f})$ adapting procedure was used with $\mathrm{A} 3$ and an automated $\Delta \mathrm{f}$ adapting technique was used with the other two animals. Both procedures used the same testing paradigm as described in section 2. When using the manual $\Delta \mathrm{f}$ adapting procedure, the decision to reduce $\Delta \mathrm{f}$ (making the task harder) was made by the experimenter. When using the automated $\Delta \mathrm{f}$ adapting procedure, this decision was made by a software algorithm. Initially the ratio between the frequency difference and reference frequency $(\Delta \mathrm{f} / \mathrm{f})$ was set to 0.6 and subsequently it was varied based on performance. If d' from 100 consecutive, in-session trials was above $1.5, \Delta \mathrm{f} / \mathrm{f}$ was halved, while a d' of below 0.5 resulted in $\Delta \mathrm{f} / \mathrm{f}$ being doubled. An upper limit of $\Delta \mathrm{f} / \mathrm{f}=0.6$ was used based on previous pilot results that suggest that this should be an easy discrimination for all animals. Figure 5A shows a psychometric function in which d' is plotted 
versus $\Delta \mathrm{f}$. Linear regression was used to find the frequency difference threshold (at d' =1) with $95 \%$ confidence intervals as shown on figure 5A. It is important to note that this analysis was made only on the linear portion of the psychometric function, thus the one point for $\Delta \mathrm{f} / \mathrm{f}=0.6$ was excluded from the regression analysis as it is located in the psychometric function's saturation region. The reference frequency was changed after performance stabilized (as assessed by the $95 \%$ confidence interval and discussed in section 3.5) which usually occurred after 2 weeks of testing.

\subsection{Performance stability}

A representative example of performance over a two week period is shown in Figure 6A. Confidence intervals in figure 6A are based on quantitative data from the day we changed the reference frequency. After day 7 , there is little change in the confidence intervals, indicating stable performance. After that time, the difference in upper and lower threshold confidence intervals stays within 10 percent of the previous session and the slope of 3 consecutive sessions is below $0.01 \Delta \mathrm{f} / \mathrm{f}$ per day.

\subsection{Repeatability}

It is important to establish how repeatable the measured thresholds are, therefore the same measurement was repeated a number of times. Figure $6 \mathrm{~B}$ shows the performance of A1, at $6 \mathrm{kHz}$ reference frequency, over a period of 14 weeks. Each measurement was based on the combined data from a 2-week test period. Overall, no change in performance was observed (figure 6B) and confidence intervals got smaller over the whole period.

\subsection{Response bias and loudness cue}

We tested whether animals did not develop any unusual strategies to achieve food delivery, but were actually performing the frequency discrimination task. One such strategy could be relying on the order of the stimuli. To confirm that that was not the case performance for different stimulus orders was compared. Hit rate for the two different orders of target stimuli presentation (low-high and high-low) was not significantly different across all animals (two-way ANOVA, Order $\times$ Animal; $\mathrm{p}=0.99$ ). FA rate for the two different orders of 
non-target stimuli presentation (low-low and high-high) was also not significantly different across all animals (two-way ANOVA, Order $\times$ Animal; $\mathrm{p}=0.84$ ).

Another strategy could be relying on a residual loudness cue. The level roving of the tones was intended to eliminate any loudness cues; however, the choice of roving extent was arbitrary and made before starting the experiment. To test that the extent of roving was sufficient and loudness cues were actually eliminated for all cats, we compared performance in four different target stimuli combinations: low-high and high-low frequencies with lower dB SPL tone first, higher dB SPL tone second, and vice versa. There was no significant effect of loudness (two-way ANOVA, Stimulus type $\times$ Animal; $p=0.63$ ), which we interpret as the animals not relying on loudness cues to perform the task.

\section{Discussion}

\subsection{Frequency discrimination thresholds}

Thresholds established by the current method shown in figure 5B are similar to those from two previously reported experiments (Brown et al., 2004, Hienz et al., 1993). Brown et al. (2004) employed a hold-release auditory association paradigm where difference thresholds were taken as 50\% performance level (calculated from a hit rate corrected for FA rate). Hienz et al. (1993) used a positive operant conditioning task, and difference thresholds were calculated as the frequency difference producing a detection score halfway between the FA rate and $100 \%$. Despite these variations in methods and definitions of threshold, the results produced by Brown et al. (2004) and Hienz et al. (1993) studies are similar to ours. In a much earlier study, Elliott et al. (1960) used avoidance-conditioning with electric shocks as reinforcement and a method of limits with a threshold determined by the experimenter based on inter- and intra-animal consistency. Their method produced lower thresholds, likely due to methodological differences such as the animals not being penalized for FAs, resulting in lower criterion allowing extremely high performance levels (an opinion shared by Brown et al. (2004)). It is also not clear how Elliott et al. (1960) regarded FA responses and what proportion of non-target trials were used. By using signal detection theory (i.e. calculating d') and presenting $50 \%$ non-target trials, we ensure that an unbiased measure of performance is made.

\subsection{Data collection times}


The literature does not provide exact figures on how long it takes to train a naive animal. Brown et al. (2004) reported that it took a few thousand trials to train animals, which is similar to the task described here. In terms of data collection times, with 50-300 trials per day, 3-5 days per week (Brown et al., 2004) it could possibly take double the time required with our method which was designed to operate continuously (150200 trials per day, 7 days per week). Elliott et al. (1960) reported that their testing sessions were limited to only 25 trials; if we assume a maximum of 1-2 sessions per day (given the need to transport animals to the dedicated testing facility), data collection time will be around 3 times slower than in current experiment. It should also be stressed that in both of these studies training and testing were supervised, imposing a high time demand on laboratory personnel.

The time it takes to train a non-naive animal on a new reference frequency (frequency generalization) is also important. Heinz et al. (1993) reported that it took 118 sessions (5 days/week in sessions lasting 45-90 min) to achieve a stable performance at $3 \mathrm{kHz}$. Assuming that 2 sessions were conducted per day, it took more than 8 weeks compared with less than 2 weeks using the method described here (figure 6A).

\subsection{Advantages and difficulties}

As psychoacoustic testing was performed in animals' home cages, rather than in a dedicated testing facility, the environment was relatively noisy. To overcome typical animal facility noise, SPL of the test tones was set higher compared with standard psychoacoustic testing. As cats were tested simultaneously, another potentially distracting sound source was generated by other cats' testing units. Since distance between feeders allowed sufficient SPL attenuation (around $20 \mathrm{~dB}$ SPL) and cats could localize the sound of their feeders (based on direct observation) they most likely were able to distinguish between tones generated by their own and other cats' feeders. The complex sound field of the animals' cages could also be expected to cause sound wave reverberations and reflections; however, sound intensity of different tones was relatively constant (within $5 \mathrm{~dB}$ SPL) around the animals' approximate head locations. Using the earlier described session concept and averaging performance measurement over 100 trials help reduce the effect of potentially distracting environmental factors. Poorer performance is expected when testing in uncontrolled sound environment, however, results are comparable with 2 out of 3 similar studies.

Based on our observations, human interaction in the testing procedure caused animal distraction thus fully automated operation was desirable. In frequency discrimination threshold testing, upper and lower d' 
conditions could be preset and $\Delta \mathrm{f} / \mathrm{f}$ decreased or increased according to animals' performance, so that minimum human interaction was necessary.

Most other psychoacoustic experiments use negative reinforcement such as electric shocks or air puffs, restraint, animal transfer, or combinations of those practices. We show that even without utilizing these practices, animals can reach comparable performance levels.

As the system can run nonstop, provided the food is replenished, and act as the main or sole food supply for the animal, there is no food deprivation after the animal has learned the task. During the training phase, some food was occasionally manually dispensed at the end of the day to ensure sufficient food intake; however, after learning the task access to food was essentially unlimited as FA rate is low and the animal controls the number of trials generated. Even with no food deprivation during testing, we show consistent performance (figure 6) with only $23 \%$ variation in trials per day (calculated from table 1 ).

\section{Summary}

A novel behavioral testing procedure was designed and tested on 7 animals. Each animal produced 150 to 200 trials per day within about 8 self initiated sessions, with no removal from original living cage or negative reinforcement such as electric shocks or air puffs. Data was stable over short periods of 1 week and repeatable over longer test periods of 14 weeks. Frequency discrimination thresholds from this and previously conducted studies are comparable.

In the future we plan to utilize the described method in chronic cochlear-implant studies so that animals with different hearing conditions could be tested and compared. Additionally, we intend to use this behavioral paradigm to test different auditory and visual detection and discrimination abilities.

\section{Acknowledgments}

This work was funded by the National Institutes of Health (HHS-N-263-2007-00053-C), the National Health and Medical Research Council of Australia and The Department of Electronic Engineering, La-Trobe University. The Bionics Institute acknowledges the support it receives from the Victorian Government through its Operational Infrastructure Support Program. The authors are grateful to Alison Neil, Nicole Critch and Amy Morley for technical assistance; Andrew Wise and Sam Irvine for advice; Sue Pierce for 
veterinary advice; Sue Mckay for animal maintenance; Dexter Irvine for comments on the earlier versions of the manuscript.

\section{References}

AMMERSDÖRFER, S., GALINSKI, S. \& ESSER, K. H. 2012. Effects of aversive experience on the behavior within a custom-made plus maze in the short-tailed fruit bat, Carollia perspicillata. Journal of Comparative Physiology A: Neuroethology, Sensory, Neural, and Behavioral Physiology, 1-7.

BALCOMBE, J. P., BARNARD, N. D. \& SANDUSKY, C. 2004. Laboratory routines cause animal stress. Journal of the American Association for Laboratory Animal Science, 43, 42-51.

BEITEL, R. E., SNYDER, R. L., SCHREINER, C. E., RAGGIO, M. W. \& LEAKE, P. A. 2000. Electrical cochlear stimulation in the deaf cat: comparisons between psychophysical and central auditory neuronal thresholds. Journal of Neurophysiology, 83, 2145-62.

BROWN, M., IRVINE, D. \& PARK, V. 2004. Perceptual learning on an auditory frequency discrimination task by cats: association with changes in primary auditory cortex. Cerebral cortex, 14, 952.

CARLSTEAD, K., BROWN, J. L. \& STRAWN, W. 1993. Behavioral and physiological correlates of stress in laboratory cats. Applied Animal Behaviour Science, 38, 143-158.

CLARK, G. M. 1998. Research advances for cochlear implants. Auris Nasus Larynx, 25, 73-87.

DUKE, J. L., ZAMMIT, T. G. \& LAWSON, D. M. 2001. The effects of routine cage-changing on cardiovascular and behavioral parameters in male Sprague-Dawley rats. Journal of the American Association for Laboratory Animal Science, 40, 17-20.

ELLIOTT, D. N., STEIN, L. \& HARRISON, M. J. 1960. Determination of absolute-intensity thresholds and frequencydifference thresholds in cats. Journal of the Acoustical Society of America, 32, 380-384.

ERNST, K., PUPPE, B., SCHÖN, P. C. \& MANTEUFFEL, G. 2005. A complex automatic feeding system for pigs aimed to induce successful behavioural coping by cognitive adaptation. Applied Animal Behaviour Science, 91, 205218.

FALLON, J. B., SHEPHERD, R. K., BROWN, M. \& IRVINE, D. R. 2009. Effects of neonatal partial deafness and chronic intracochlear electrical stimulation on auditory and electrical response characteristics in primary auditory cortex. Hearing Research, 257, 93-105.

HEEGER, D. 1997. Signal detection theory. New York: New York University.

HEFFNER, R. S. \& HEFFNER, H. E. 1985. Hearing range of the domestic cat. Hearing Research, 19, 85-88.

HIENZ, R. D., SACHS, M. B. \& ALESZCZYK, C. M. 1993. Frequency discrimination in noise: comparison of cat performances with auditory-nerve models. Journal of the Acoustical Society of America, 93, 462-9.

KOOT, S., ADRIANI, W., SASO, L., VAN DEN BOS, R. \& LAVIOLA, G. 2009. Home cage testing of delay discounting in rats. Behavior Research Methods, 41, 1169-1176.

LIU, Y., QIN, L., ZHANG, X., DONG, C. \& SATO, Y. 2010. Neural correlates of auditory temporal-interval discrimination in cats. Behavioural Brain Research, 215, 28-38.

MAY, B. J., HUANG, A. Y., ALESZCZYK, C. M. \& HIENZ, R. D. 1995. Design and Conduct of Sensory Experiments for Domestic Cats. In: KLUMP, G. M. (ed.) Biomethods: Methods of Comparative Acoustics. Basel: Birkhäuser Verlag.

PRETORIUS, L. L. \& HANEKOM, J. J. 2008. Free field frequency discrimination abilities of cochlear implant users. Hearing Research, 244, 77-84.

RECANZONE, G. H., JENKINS, W. M., HRADEK, G. T. \& MERZENICH, M. M. 1991. A behavioral frequency discrimination paradigm for use in adult primates. Behavior research methods, instruments \& computers, 23, 357-369.

SLOAN, A. M., DODD, O. T. \& RENNAKER II, R. L. 2009. Frequency discrimination in rats measured with tone-step stimuli and discrete pure tones. Hearing Research, 251, 60-69.

SMITH, D. W., FINLEY, C. C., VAN DEN HONERT, C., OLSZYK, V. B. \& KONRAD, K. E. M. 1994. Behavioral and electrophysiological responses to electrical stimulation in the cat: I. Absolute thresholds. Hearing Research, 81, 1-10.

TONG, Y. C., CLARK, G. M., BLAMEY, P. J., BUSBY, P. A. \& DOWELL, R. C. 1982. Psychophysical studies for two multiple-channel cochlear implant patients. Journal of the Acoustical Society of America, 71, 153-60. 
VOLLMER, M., BEITEL, R. E. \& SNYDER, R. L. 2001. Auditory detection and discrimination in deaf cats: psychophysical and neural thresholds for intracochlear electrical signals. Journal of Neurophysiology, 86, 2330.

WILSON, B. S. \& DORMAN, M. F. 2008. Cochlear implants: A remarkable past and a brilliant future. Hearing Research, 242, 3-21. 
Figures and Tables:
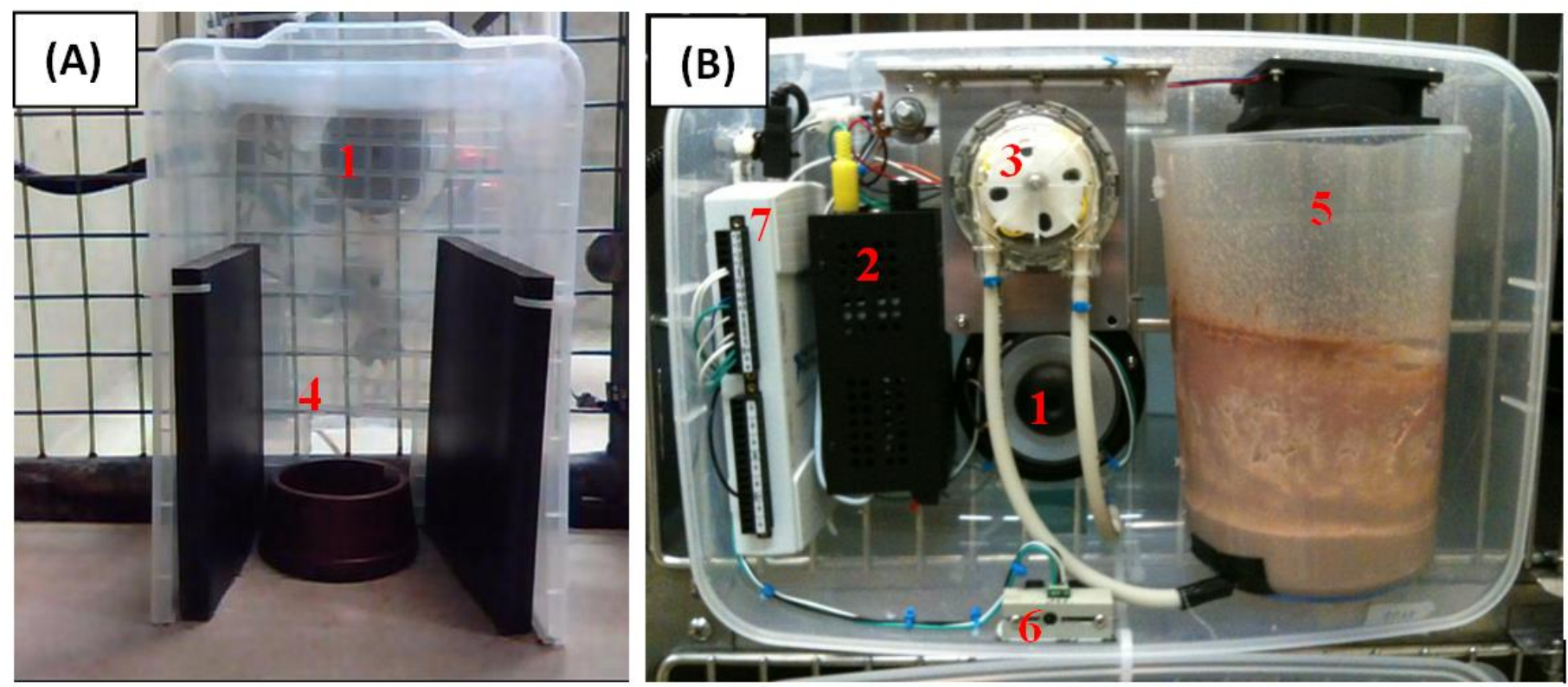

Figure 1. The front of the system as viewed by the animal is shown in (A). Various system components

located outside the animal's enclosure are shown in (B). 1, Speaker; 2, Audio amplifier; 3, Peristaltic pump;

4, Two light sources and food delivery opening; 5, Food tank and stirrer; 6, Proximity sensor; 7, Data acquisition D/A device.

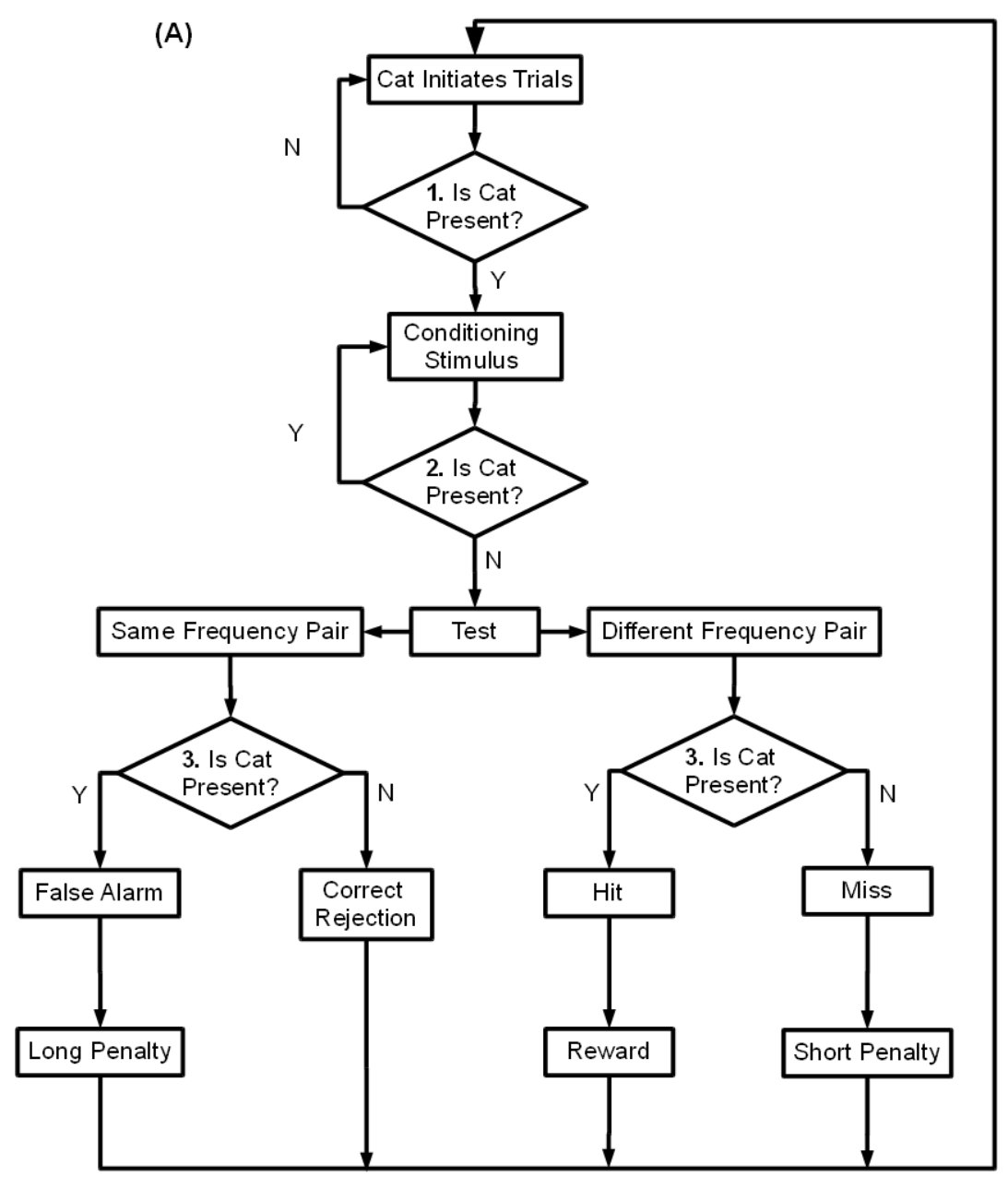

(B)

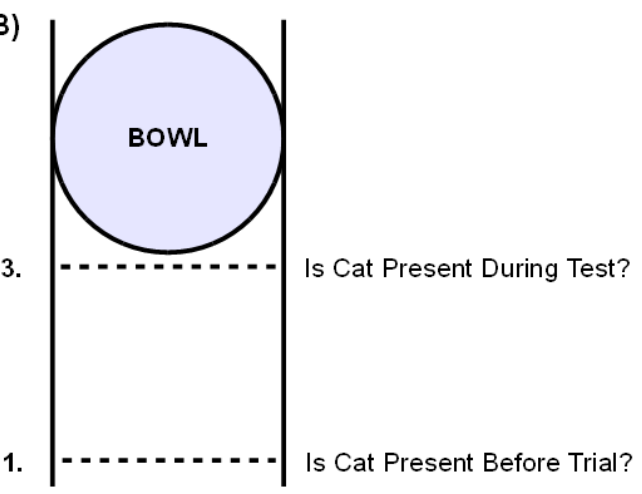

2. Is Cat Present Before Test? 
Figure 2. System operation: (A) Flow chart describing a single trial procedure. (B) Three different sensing distances utilized at different stages during a single trial.
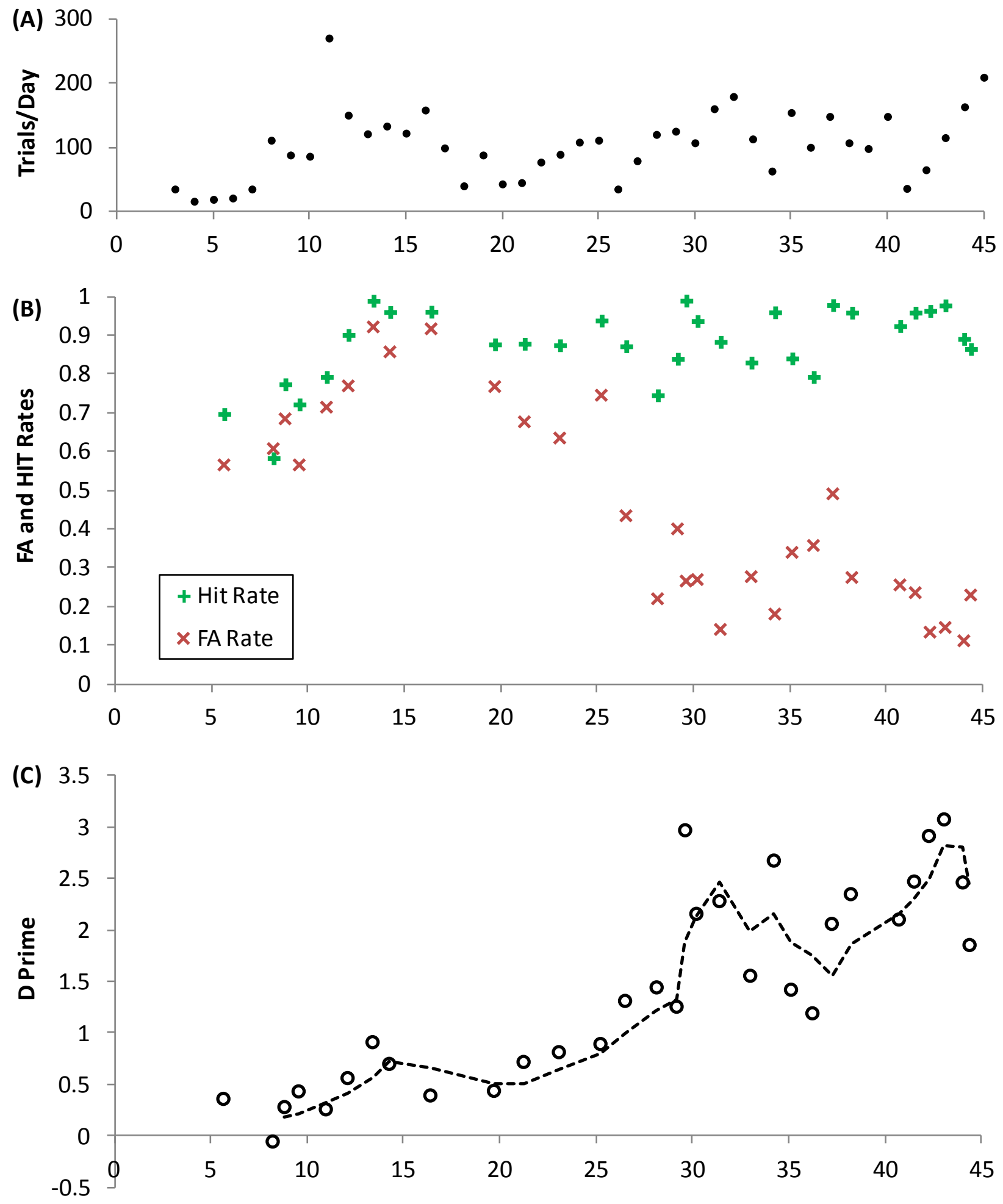

Day Number

Figure 3. Typical learning curve (cat B1) over first 45 days with all parameters kept constant (reference frequency equal to $8.5 \mathrm{kHz}$ and the ratio between the frequency difference and reference frequency equal to 0.6). (A) Trials per day throughout the 45 day period. (B) Green plus signs and red crosses represent hit and 
FA (false alarm) rate, respectively, which were calculated using 100-trial windows. (C) Variation in d-prime (d') over training period. Dashed line represents 3-point interpolation.

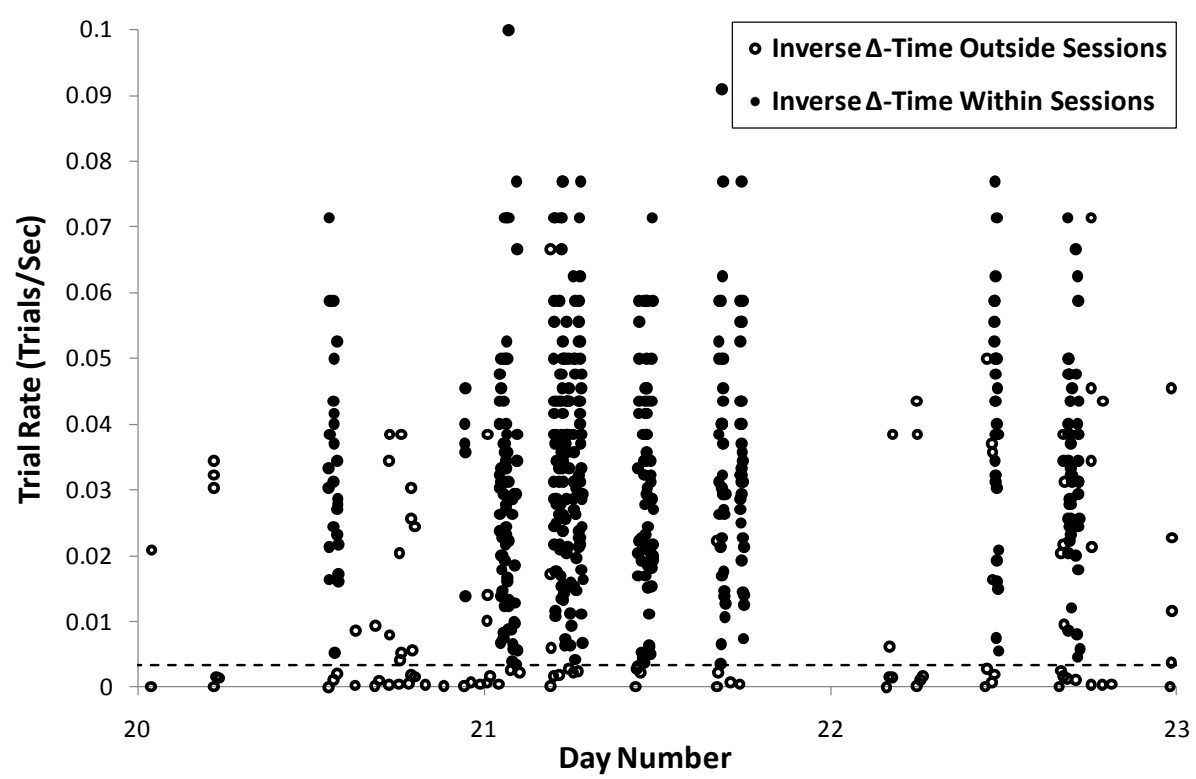

Figure 4. Sample trial rate over 3 days from one animal. Closed and open circles represent trials classified as "in-session" and "out-of-session" respectively. The dotted line represents a value of 0.003 trials/s which indicates 5 min between trials.

\begin{tabular}{|c|c|c|c|c|c|c|}
\hline Cat & $\begin{array}{l}\text { Age } \\
\text { (months) }\end{array}$ & $\begin{array}{c}\text { Training } \\
\text { period } \\
\text { (days) }\end{array}$ & Trials per day & $\begin{array}{c}\text { Trials per } \\
\text { session }\end{array}$ & $\begin{array}{c}\text { Sessions per } \\
\text { day }\end{array}$ & $\begin{array}{c}\text { Out-of- } \\
\text { session trials } \\
(\%)\end{array}$ \\
\hline A1 & 6 & 16 & $195(25)$ & $13(9)$ & $10.3(3.15)$ & 25.6 \\
\hline $\mathrm{A} 2$ & 6 & 13 & $203(50)$ & $19.7(9.4)$ & $8.7(2.8)$ & 11.9 \\
\hline A3 & 19 & - & $173.1(40.5)$ & $17.8(14.2)$ & $7.85(2.6)$ & 19.7 \\
\hline B1 & 5 & 30 & $113(31)$ & $19(14.5)$ & $4.4(1.5)$ & 22.5 \\
\hline $\mathrm{B} 2$ & 6 & 33 & $182(40.6)$ & $13.2(7.25)$ & $10.3(2.7)$ & 20.9 \\
\hline B3 & 13 & 29 & $201.7(27.8)$ & $21.2(10.7)$ & $9(1.6)$ & 8 \\
\hline B4 & 7 & - & $214.8(76)$ & $15.8(11)$ & $9.1(3.6)$ & 22.1 \\
\hline
\end{tabular}

Table 1. Animal information and behavioral statistics. Numbers in brackets indicate standard deviations.

Training period indicates the number of days it took each cat to reach $\mathrm{d}^{\prime}=2$ from initial introduction to the training equipment. Trials per day, trials per session, sessions per day and out-of-session trials were taken from a week's worth of data after animal's performance was well above chance (d'>2). As animals A3 and B4 were used for various other procedures during training, their training periods were excluded. 

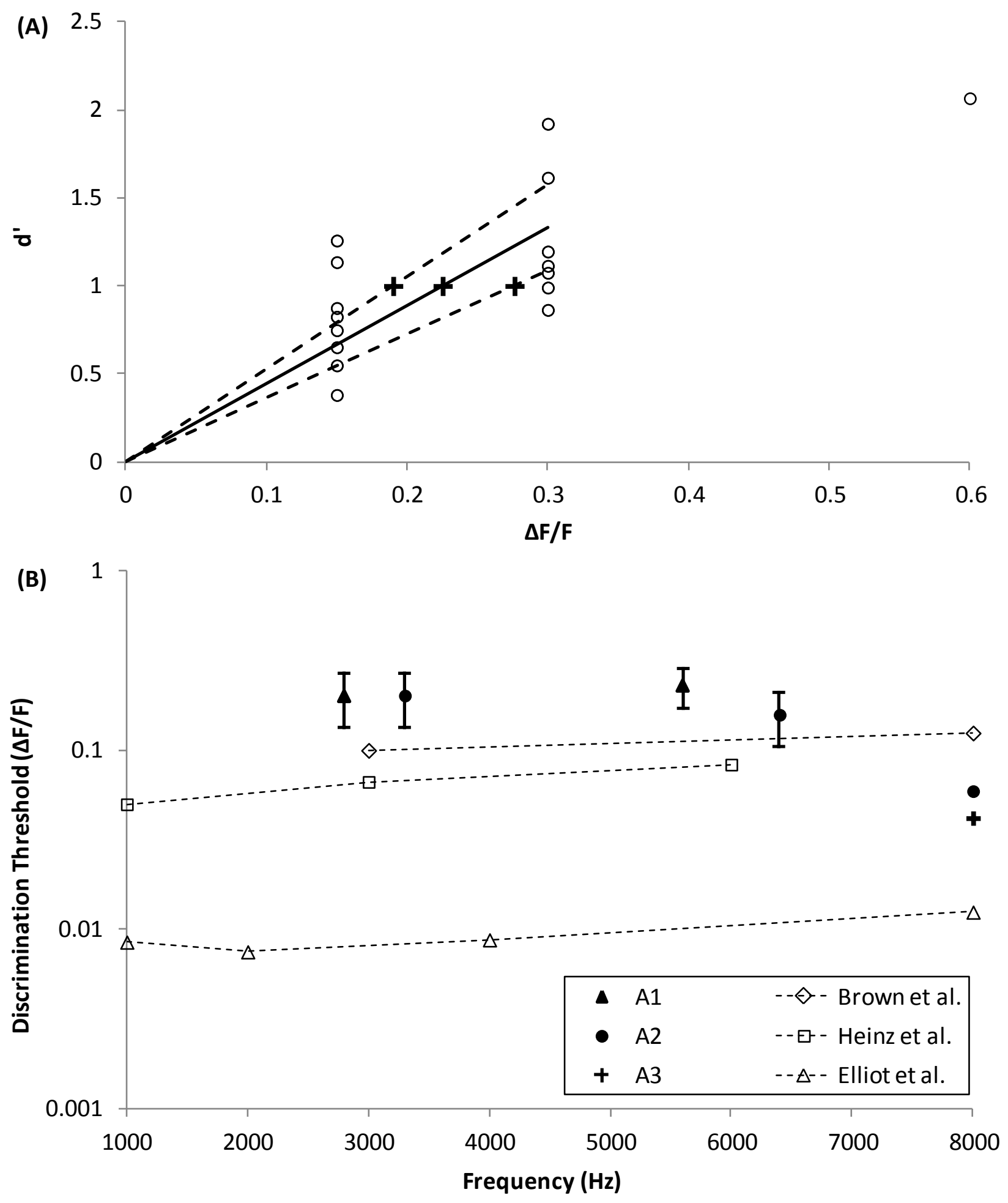

Figure 5. (A) Psychometric function of cat A1 at $6 \mathrm{kHz}$. Straight line is linear regression of 15 data points for $\Delta \mathrm{f} / \mathrm{f}$ values of $0.3,0.15$, constrained to include a d' of zero at $\Delta \mathrm{f} / \mathrm{f}$ equal to zero. Dotted lines indicate 95\% confidence intervals. Cross signs indicate the threshold estimate ( $\left.\mathrm{d}^{\prime}=1\right)$ and its confidence interval. (B) Psychoacoustic frequency discrimination thresholds of three animals in the current study. Two animals were used to establish a threshold at each reference frequency. Thresholds for A1 and A2 were determined 3 times at 3 and $6 \mathrm{kHz}$, and thresholds for A2 and A3 were determined once at $8 \mathrm{kHz}$. Error bars are standard error 
of the mean. Data sets connected by lines are taken from the results in three previous reports. (Brown et al., 2004, Hienz et al., 1993, Elliott et al., 1960).
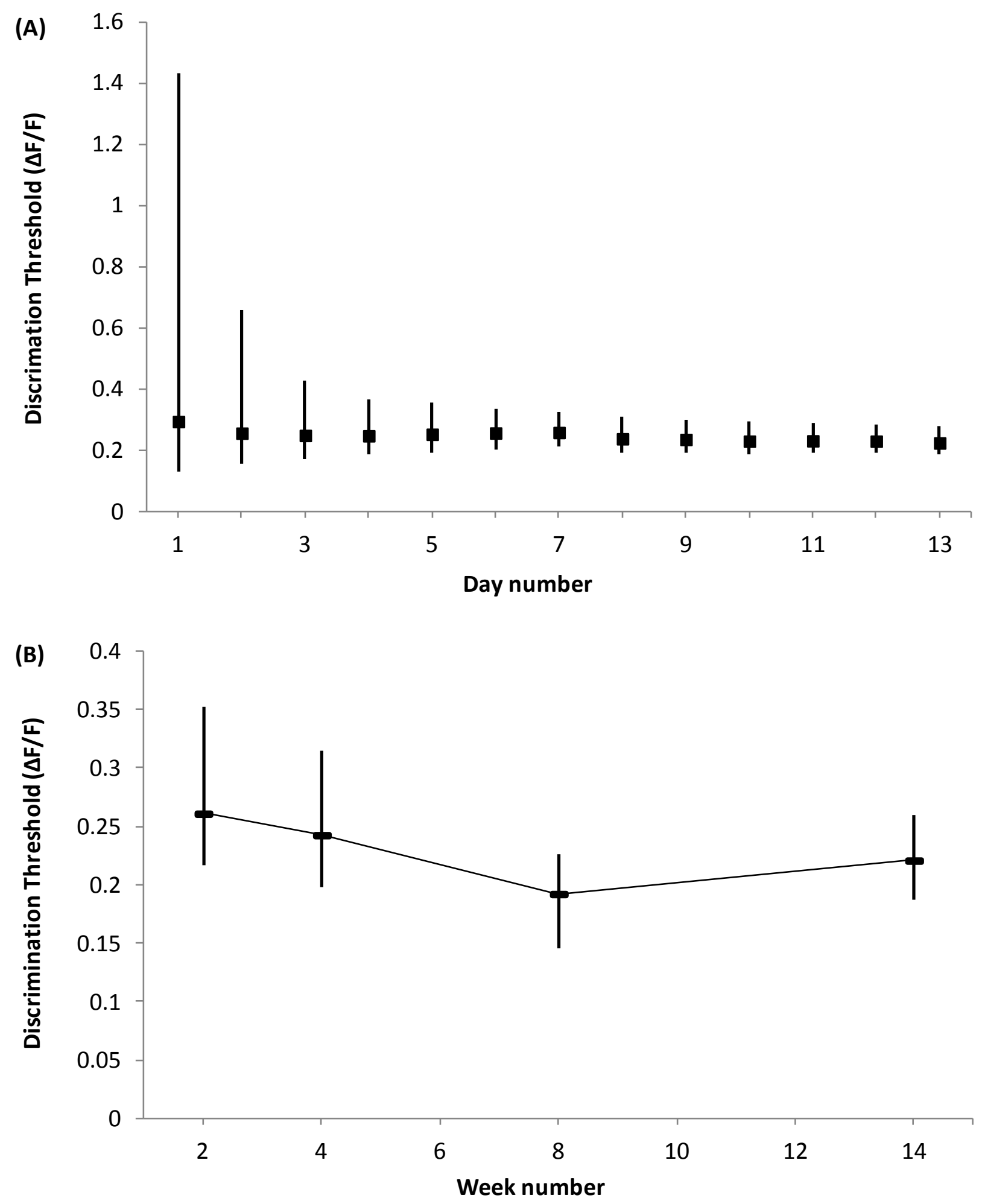

Figure 6. Performance stability and repeatability for cat A1 using adaptive procedure to vary $\Delta \mathrm{f} / \mathrm{f}$ while reference frequency held constant at $6 \mathrm{kHz}$. (A) Discrimination threshold stability over 2 weeks. Squares represent the means and error bars indicate confidence intervals. (B) Discrimination threshold repeatability 
in 4 test sessions over 14-week period. Each data point represents two weeks of data. Error bars indicate 95\% confidence intervals. 


\section{University Library}

\section{- M M I N E R VA A gateway to Melbourne's research publications}

Minerva Access is the Institutional Repository of The University of Melbourne

Author/s:

Benovitski, YB;Blamey, PJ;Rathbone, GD;Fallon, JB

Title:

An automated psychoacoustic testing apparatus for use in cats

Date:

2014-03-01

Citation:

Benovitski, Y. B., Blamey, P. J., Rathbone, G. D. \& Fallon, J. B. (2014). An automated psychoacoustic testing apparatus for use in cats. HEARING RESEARCH, 309, pp.1-7. https://doi.org/10.1016/j.heares.2013.11.002.

Persistent Link:

http://hdl.handle.net/11343/43088 